\title{
TRIAGEM VIRTUAL PARA A SELEÇÃO DE CANDIDATOS A INIBIDORES DA DIIDROOROTATO DESIDROGENASE DE PLASMODIUM FALCIPARUM
}

\author{
Moysés Fagundes de Araujo Neto ${ }^{1}$; Franco Henrique Andrade Leite ${ }^{2}$ \\ 1. Bolsista PIBIC/CNPq, Graduando em Farmácia, Universidade Estadual de Feira de Santana, e-mail: \\ moysesfagundes@gmail.com \\ 2. Orientador, Departamento de Saúde, Universidade Estadual de Feira de Santana, e-mail: fhpharm@gmail.com
}

PALAVRAS-CHAVE: Malária; Modelo farmacofórico; Plasmodium falciparum.

\section{INTRODUÇÃO}

A malária é uma doença infecciosa causada por protozoários do gênero Plasmodium spp., sendo o P. falciparum responsável pela forma mais severa da doença, a cerebral. A terapia farmacológica para o tratamento de pacientes com malária é ineficaz, complexa (utilização de mais de um medicamento concomitante) e possui um perfil severo de efeitos adversos (complicações gastrintestinais, tontura e efeitos psicológicos) (Leite et al., 2014; França; Santos; Figueroa-Villar, 2008), o que torna emergencial a busca por novos fármacos. Uma forma de alcançar esse objetivo é através da priorizarização de potenciais moléculas bioativas frente a alvos validados do parasito com o auxílio de ferramentas computacionais baseadas na estrutura de inibidores conhecidos, como modelos farmacofóricos e similaridade química. A triagem virtual baseada nessas técnicas tem se mostrado superiores aos ensaios randômicos na identificação de novos ligantes (Chen, 2015). Por esse motivo, uma triagem virtual hierárquica por modelo farmacofórico e similaridade química foi aplicada para a seleção de moléculas candidatas presentes no banco de produtos naturais da UEFS (NatProDB; http://natprodb.uefs.br).

\section{MATERIAL E MÉTODOS}

Seleção dos inibidores: Um conjunto de 40 inibidores triazolopirimidínicos, com valor de atividade biológica $\left(\mathrm{IC}_{50}=0,019-0,93 \mu \mathrm{M}\right)$ foram coletados da literatura (Coteron, 2011) e posteriormente divididos em treino $(n=28)$ e teste $(n=12)$ para a geração e avaliação dos modelos farmacofóricos, respectivamente. As estruturas 2D foram obtidas no programa Marvin ${ }^{\circledR}$ Sketch 15.4.20 (Chemaxon, 2015, https://www.chemaxon.com/) e convertidas para o formato 3D na plataforma SYBYL®-X 2.0 (Tripos, 2010). Em seguida, as cargas parciais foram calculadas pelo método Gaisteiger-Huckel e as estruturas foram minimizadas por gradiente conjugado com um critério de convergência de $0,001 \mathrm{Kcal} / \mathrm{mol}$, utilizando campo de força Tripos conforme disponível na plataforma SYBYL®-X 2.0.

Geração dos modelos farmacofóricos: Os modelos farmacofóricos foram gerados em duas etapas: Inicialmente, as conformações dos inibidores foram obtidas através de Algoritmo Genético implementado no módulo GALAHAD, no qual o tamanho da população e o número máximo de gerações para os inibidores da PfDHODH foram ajustados para 150 e 40 . Os demais parâmetros (CRUZAMENTO = 1,0 e MUTAÇÃO $=1,0$ ) foram mantidos nos seus valores padrão. Posteriormente, os confôrmeros foram alinhados entre si para a construção dos modelos farmacofóricos.

Avaliação dos modelos farmacofóricos: Inicialmente, os modelos farmacofóricos foram avaliados quanto aos parâmetros estatísticos internos do GALAHAD (ENERGY < 100 $\mathrm{Kcal} / \mathrm{mol}$ e PARETO $\neq 00$ ), A seguir, os modelos remanescentes foram testados quanto a habilidade de diferenciar ativos de falsos positivos (decoys) construídos com o auxílio do servidor DUD-E numa proporção de 1:50 (Mysinger, et al. 2012). O banco contendo todas as moléculas (ativos + falso positivo) foi alinhado com os modelos remanescentes para a geração da curva ROC (do inglês, Receiver Operating Characteristic) e cálculo da AUC (do inglês, area under the curve). Os modelos farmacofóricos com AUC $>0,70$ foram selecionados e 
posteriormente submetidos ao cálculo do valor da discriminação aprimorada de Boltzmann da curva ROC (do inglês, Boltzmann-Enhanced Discrimination of ROC - BEDROC) através do servidor online ROCKER (Lätti; Niinivehmas; Pentikäinen, 2016). Por fim, o modelo com melhor valor de BEDROC $(>0,50)($ alfa $=16,1)$ foi utilizado na etapa de triagem virtual do banco de moléculas NatProDB.

Priorização de potenciais inibidores: Buscando moléculas que compartilham o mesmo modo de atividade biológica, foi realizado o estudo de similaridade química pelo módulo UNITY implementado na plataforma SYBYL com as estruturas filtradas utilizando como moldes de comparação os inibidores empregados na construção dos modelos, portanto foi empregado um Índice de Tanimoto igual a 0,65. Foram calculados os principais descritores físico-químicos e topológicos através do programa Marvin Sketch 15.4.20 (Chemaxon, 2015), os quais predizem sobre a absorção e permeabilidade de moléculas por via oral através do número de aceptores de ligação de hidrogênio $(\leq 10)$ e grupos doadores de ligação de hidrogênio $(\leq 5)$, massa molecular $(\leq 500 \mathrm{~g} / \mathrm{mol})$, coeficiente de partição octanol-água (clogP) $(\leq 5)$ e da área de superfície polar (PSA) ( $\leq 140$ angstrons). Foram eliminadas as estruturas com penalidade em algum desses descritores.

\section{RESULTADOS E/OU DISCUSSÃO}

A busca por fármacos antimaláricos é crucial para o controle da doença haja vista que não existe tratamento efetivo nem vacinas. Por esse motivo, a aplicação de métodos computacionais tem possibilitado uma taxa de enriquecimento superior aos métodos randômicos. Dentre essas técnicas, os modelos farmacofóricos merecem destaque, pois são capazes de identificar requisitos estéreo-eletrônicos essenciais para inibição de alvos específicos e, portanto, são uteis na priorização de moléculas candidatas. Por esse motivo, 10 modelos farmacofóricos foram gerados e após a avaliação dos parâmetros estatísticos internos apenas oito modelos foram testados quando a taxa de acerto para a identificação de inibidores verdadeiros (Figura 1).

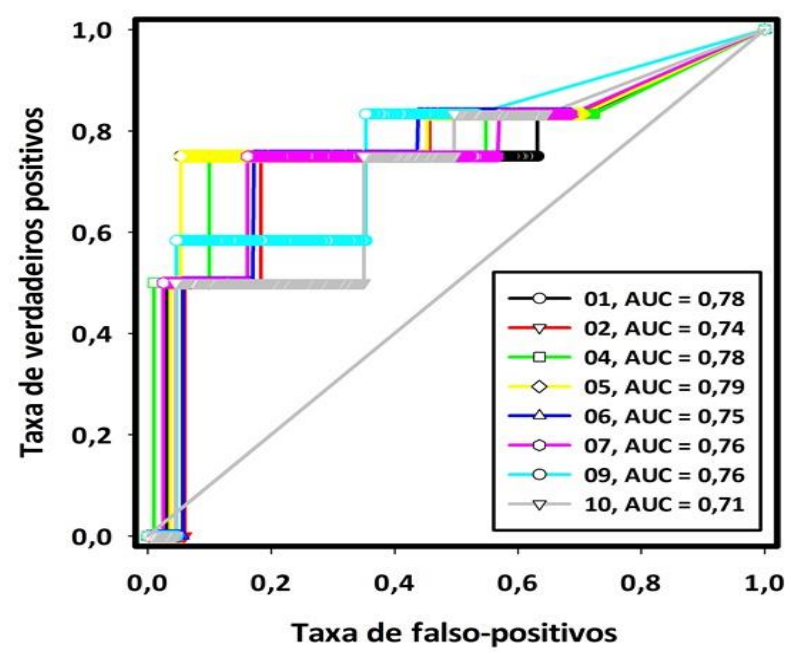

Figura 1: Curvas ROC obtidas para os modelos farmacofóricos remanescentes. A linha diagonal representa um modelo que não seria melhor do que um ensaio randômico $(\mathrm{AUC}<0,5)$.

Apesar de todos os modelos serem moderadamente preditivos (AUC > 0,7), a curva ROC esta sujeita a um poder de discriminação tardio, portanto foi empregado uma métrica capaz de avaliar a capacidade de reconhecimento precoce dos modelos. Nesta perspectiva, apenas o modelo $04(\mathrm{BEDROC}=0,51)$ foi considerado como adequado para a triagem virtual. A fim de caracterizar o modelo, uma sobreposição do inibidor potente (Figura 2 A) e pouco potente (Figura $2 \mathrm{~B}$ ) foi realizada. 


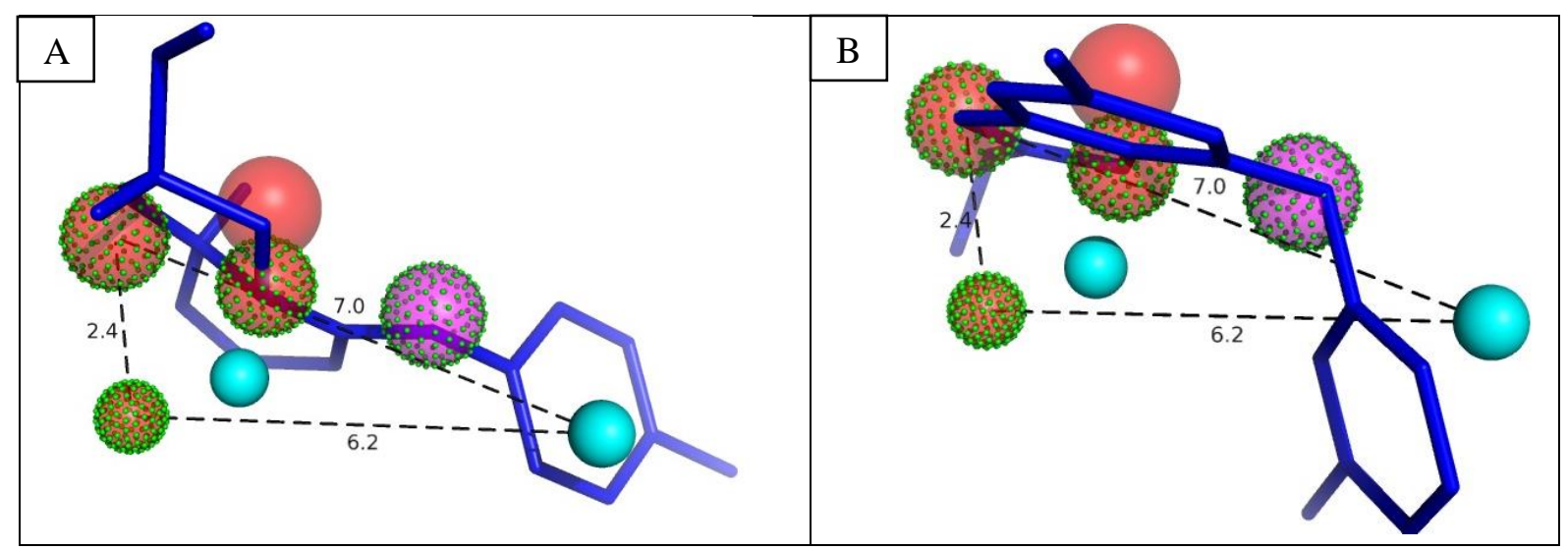

Figura 2: Sobreposição de um inibidor potente $\left(\mathrm{A}, \mathrm{IC}_{50}=0,28 \mu \mathrm{M}\right)$ e um inibidor fraco $\left(\mathrm{B}, \mathrm{IC}_{50}=6,1 \mu \mathrm{M}\right)$ no modelo 04 . Esferas em ciano representam centros hidrofóbicos (HY), pontos verdes representam doadores de ligação de hidrogênio (HBD), esferas vermelhas representam centros positivos de nitrogênio (NP) e esferas magenta representam aceptores de ligação de hidrogênio (HBA). O tamanho das esferas varia de acordo com o raio de tolerância calculado pelo GALAHAD. Todas as distâncias foram mensuradas em angstrons.

Este modelo possui um aceitador de ligação de hidrogênio (HBA), quatro doadores de ligação de hidrogênio (HBD), dois centros hidrofóbicos (HY) e quatro centros positivos de nitrogênio (NP), sendo as características HY e HBD centradas no núcleo triazolopirimidínico. Estudos conduzidos por Zhu (2015) tem demostrado que os inibidores potentes frente à PfDHODH possuem esses grupos, enquanto que na sobreposição do inibidor fraco é observado um alinhamento parcial da estrutura com o modelo, com apenas as características HBA e NP centradas no núcleo base para a inibição.

Após a triagem virtual 11 moléculas do banco de produtos naturais NatProDB foram selecionadas com base no grau de sobreposição as características do modelo (QFIT > 66,14) $(\mathrm{M}+2 \mathrm{x}$ DESVPAD), o que demostra que estas possuem alguns dos requisitos essenciais para a inibição e precisam ser investigadas. Foi detectado uma dissimilaridade entre as moléculas filtradas e os inibidores utilizados para comparação (IT < 65\%), o que as tornam potenciais moléculas bioativas com quimiotipos ainda não associados a inibição deste alvo. No entanto, a presença de requisitos para a inibição não garante que as moléculas possuirão propriedades físico-químicas para alcançar o alvo e/ou biodisponibilidade pela via oral. Portanto, foram calculados os principais descritores físico-químicos e topológicos. Com base na predição teórica empregada, sugere-se que nove das 11 moléculas filtradas, possivelmente não possuirão problemas de biodisponibilidade oral, (Tabela 1).

Tabela 1: Esqueleto químico das moléculas que demostraram possuir requisitos essenciais para a inibição da PfDHODH e preditiva biodisponibilidade por via oral

\begin{tabular}{|c|c|c|c|}
\hline Nome & VEOGCAOSF & VEOHLAOSI & VEOLLAOSI \\
\hline Estrutura 2D & & & \\
\hline QFTI & 80,69 & 84,98 & 66,50 \\
\hline Nome & VEOGTA0AF & VE0IOA0AF & VE0SJA0SI \\
\hline Estrutura 2D & & & \\
\hline QFIT & 74,00 & 72,03 & 74,14 \\
\hline
\end{tabular}




\begin{tabular}{|c|c|c|c|}
\hline Nome & VE0HDA0SF & VEOJOAOAF & VEOTLAOAF \\
\hline Estrutura 2D & & & \\
\hline QFIT & 70,30 & 72,03 & 66,86 \\
\hline
\end{tabular}

Nesta perspectiva, as estruturas selecionadas na etapa de triagem virtual demonstraram ser preditivas tanto para a inibição da PfDHODH quanto para uma biodisponibilidade oral favorável e, portanto, devem ser priorizadas em ensaios subsequentes do desenvolvimento racional de fármacos, como estudos ensaios enzimáticos.

\section{CONSIDERAÇÕES FINAIS}

A utilização do alinhamento molecular flexível pelo módulo GALAHAD foi capaz de identificar os requisitos farmacofóricos HBA e NP centradas no núcleo base triazolopirimidínico como relacionados ao reconhecimento pelo alvo, enquanto as características HY e HBD predizem a potencia do inibidor. Portanto, a presença destes requisitos é fundamental para o reconhecimento de moléculas potentes frente a PfDHODH. Dessa forma, a triagem virtual permitiu a filtragem de 11 moléculas com os requisitos essenciais de atividade biológica e potência frente ao alvo no banco de produtos naturais NatProDB. O emprego do ensaio por similaridade química despertou a perspectiva de identificação novos quimiotipos bioativos frente à $P f D H O D H$, contribuindo para a descoberta de moléculas com atividade antimaláricas dissimilares dos quimiotipos usuais. Adicionalmente, a predição teórica da biodisponibilidade oral destas estruturas está correlacionada com a priorização de inibidores da PfDHODH e com o sucesso dos ensaios subsequentes, guiando a priorização de potenciais compostos com atividade antimalárica.

\section{REFERÊNCIAS}

CHEMAXON. 2015. Marvin Sketch version 5.12.1.

COTERON, J. M. et al. 2011. Structure-Guided Lead Optimization of Triazolopyrimidine Ring Substituents Identifies Potent Plasmodium falciparum Dihydroorotate Dehydrogenase Inhibitors with Clinical Candidate Potential. Journal of Medicinal Chemistry. 54: 5540-5561. FRANCA, T. C. C.; M. G. DOS SANTOS; J. D. FIGUEROA-VILLAR. 2008. Malaria: Historical aspects and chemoterapy. Química Nova. 31 (5): 1271-1278

LÄTTI, S.; S. NIINIVEHMAS; O. T. PENTIKÄINEN. 2016. Rocker: Open source, easy-touse tool for AUC and enrichment calculations and ROC visualization. Journal of Cheminformatics. 8: 45.

LEITE, F. H. A.; A. L. FONSECA.; R. R. NUNES.; M. COMAR JÚNIOR.; F. P. VAROTTI.; A. G. TARANTO. 2014. Malaria: From old drugs to new molecular targets. Biochemistry and Biotechnology Reports. 2 (4): 59-76.

MYSINGER, M. M.; CARCHIA, M.; IRWIN, J. J.; SHOICHET, B. K. 2012. DUD-E server. J. Med. Journal of Medicinal Chemistry.

TRIPOS, Inc. 2012. SYBYL-x 2.0. Discovery Software for Computational Chemistry and Molecular Modelling.

ZHU, J. et al. 2015. Design, Synthesis, X-ray Crystallographic Analysis, and Biological Evaluation of Thiazole Derivatives as Potent and Selective Inhibitors of Human Dihydroorotate Dehydrogenase. 